\title{
ANÁLISIS COMPARATIVO ENTRE UN JARABE DE TUNA (OPUNTIA AMYCLAEA T.) Y UN JARABE COMERCIAL SABOR MAPLE
}

\section{COMPARATIVE ANALYSIS BETWEEN PRICKLY PEAR SYRUP (OPUNTIA AMYCLAEA T.)} AND A COMMERCIAL MAPLE FLAVOR SYRUP

\author{
Juan José Aguilar Lugo Marino*, Sendy Janet Sandoval Trujillo**, \\ Eva Rossina Aguilar Lugo Gerez ${ }^{\star \star \star}$
}

* Doctor en Administración. Universidad Autónoma del Estado de Hidalgo. Orcid.org/0000-0003-15742990. Abasolo No. 600 Col. Centro C.P. 42000 Pachuca, Hidalgo. México.

** Maestría en Impuestos. Universidad Autónoma del Estado de México. E-mail: sjsandovalt@uaemex.mx *** P.I.A. Universidad Autónoma del Estado de Hidalgo. E-mail: evarossina66@hotmail.com

Dirección para recibir correspondencia: juanjoseaguilarlugo@yahoo.com.mx 
ANÁLISIS COMPARATIVO ENTRE UN JARABE DE TUNA (OPUNTIA AMYCLAEA T.) Y UN JARABE COMERCIAL SABOR MAPLE

\section{RESUMEN}

En los años de alta producción de tuna suele perderse hasta un $60 \%$ de la cosecha, debido principalmente a problemas de comercialización y en la citada comercialización el mayor costo, el $40 \%$ es en su almacenamiento; los meses de agosto y septiembre son los de mayor producción y por la sobre oferta se llega a vender hasta un $50 \%$ por debajo de su valor, ya que su vida pos cosecha varía de nueve a quince días y en ese tiempo el producto tiene que comercializarse. Por tal motivo, el objetivo de la presente investigación es crear un producto innovador a base de tuna y que tenga la aceptación por sus características organolépticas con semejanza al jarabe sabor maple que hay en el mercado.

OBJETIVO: Crear un producto innovador a base de tuna y que tenga la aceptación por sus características organolépticas con semejanza al jarabe sabor maple que hay en el mercado.

MATERIAL Y MÉTODO: Para conocer su aceptación y en qué línea de productos ubicarlo se realizó un análisis comparativo de degustación entre ambos productos sobre tres dimensiones: sabor, consistencia y color; recurriendo a la $t$ de student que se utiliza para muestreos apareados (relacionados) menores a 30 panelistas. Para medir su fiabilidad, se utilizó el coeficiente de Alfa de Cronbach.

RESULTADOS: Los panelistas si asocian el producto desarrollado con el comercial dentro de los jarabes de sabores, por lo que se sugiere posicionarlo dentro de esta línea de productos. Respecto a la aceptación ésta existió en dos (sabor y consistencia) de las tres dimensiones.

CONCLUSIONES: Al existir aceptación en dos de las tres dimensiones (sabor y consistencia), se sugiere trabajar en la dimensión faltante de aceptar (color), al desarrollar el nuevo producto con otras tonalidades y posteriormente conocer su grado de aceptación.

PALABRAS CLAVE: Tuna. Jarabe de sabores. Posicionamiento de nuevos productos. 
ANÁLISIS COMPARATIVO ENTRE UN JARABE DE TUNA (OPUNTIA AMYCLAEA T.) Y UN JARABE COMERCIAL SABOR MAPLE

\section{ABSTRACT}

In the years of high prickly pear production usually $60 \%$ of the harvest is lost mainly to commercialization problems, and in this commercialization, the highest cost, $40 \%$, is its storage; the months of August and September are those of greater production and because of the oversupply it is possible to sell this product up to $50 \%$ below its value, since its post-harvest life varies from nine to fifteen days and in that time the product has to be commercialized. For this reason the objective of this research is to create an innovative product based on prickly pear and that has the acceptance due to its organoleptic characteristics similar to the maple flavor syrup that is on the market.

OBJECTIVE: to create an innovative product based on prickly pear and that has the acceptance due to its organoleptic characteristics similar to the maple flavor syrup that is on the market.

MATERIAL AND METHOD: To know its acceptance and in which line of products can be located, a comparative analysis of tasting between both products was carried out on three dimensions: taste, consistency and color; using Student's t test, which is used for paired (related) samples of less than 30 panelists. To measure its reliability, the Cronbach's Alpha coefficient was used.

RESULTS: The panelists do associate the developed product with the commercial one within the flavored syrups, so it is suggested to place it within this product line. Regarding the acceptance, this existed in two (flavor and consistency) of the three dimensions.

CONCLUSIONS: When there is acceptance in two of the three dimensions (flavor and consistency), it is suggested to work in the missing dimension (color) when developing the new product with other tonalities and later to know its level of acceptance

KEY WORDS: Prickly pear. Flavored syrup. Positioning of new products. 
ANÁLISIS COMPARATIVO ENTRE UN JARABE DE TUNA (OPUNTIA AMYCLAEA T.) Y UN JARABE COMERCIAL SABOR MAPLE

\section{INTRODUCCIÓN}

\section{Antecedentes de la producción de tuna}

El nopal es una planta propia del paisaje mexicano y uno de los símbolos más importantes de nuestra República Mexicana. Se produce en 27 entidades federativas del país, destacando la Ciudad de México, Estado de México, Morelos, San Luis Potosí, Jalisco, Aguascalientes, Hidalgo y Puebla; que aportan casi el 95\% de la producción nacional (Anaya-Pérez \& BautistaZane, 2008, p. 167).

Junto con el maíz (Zea mays, L.), el frijol (Phaseolus vulgaris, L.) y el maguey (Agave americana), fue alimento fundamental, y responsable en buena medida de asentamientos humanos y del desarrollo cultural de grupos chichimecas del centro y del norte del país. También trasciende su utilización como bebida, tinte, medicina, entre otros usos. Fue hasta la segunda mitad del siglo XX, cuando el gobierno mexicano e instituciones educativas empezaron a dar importancia al cultivo del nopal y su complemento en dietas alimenticias (Ibídem, p. 168).

El rescatar la cultura del nopal y la tuna es coadyuvar al aprovechamiento integral y sustentable que se ha ido perdiendo, ya que existe una baja importancia económica del nopal (tuna, nopalito y forraje), determinada por los bajos volúmenes comercializados debido al alto nivel de intermediarismo (Méndez \& García, 2006, p. 4), lo que ha propiciado la falta de información actualizada que permita mostrar su importancia socioeconómica y ambiental, así como mecanismos para mejorar los canales de comercialización directos.

En el aspecto ambiental, Anaya-Pérez \& Bautista-Zane (Op. Cit., p. 168) dicen que: "se ha promovido la plantación para industrializarlo (el nopal) y para reforestar amplias áreas sin vegetación, buscando con ello controlar la erosión de los suelos".

La tuna resulta también ser un alimento con algunos nutrientes dignos de tomarse en cuenta como son: fibra total (0.42 a 0.68\%), proteína (0.39 a 0.57\%), magnesio, calcio, fósforo y zinc; así como trazas de hierro y potasio (Tomás, et. Al., 2012: 70), así como vitamina C, vitamina E y una propiedad funcional muy importante que es la actividad antioxidante, ya que los antioxidantes naturales han despertado el interés para emplearlos como sustitutos de los antioxidantes sintéticos (Sumaya, Suárez, Cruz, Alanís \& Sampedro, 2010, pp. 436-438), quizá los valores se ven muy reducidos, pero es importante mencionar que éstos fueron tomados del 
ANÁLISIS COMPARATIVO ENTRE UN JARABE DE TUNA (OPUNTIA AMYCLAEA T.) Y UN JARABE COMERCIAL SABOR MAPLE

análisis del fruto de manera integral, pero si vemos los sólidos totales de la tuna que son de 11,23\% al 13.08\% (Tomás, Op Cit., p. 73) el porcentaje de nutrientes en base a sólidos totales se eleva casi 10 veces. Con lo que respecta a otro elemento que es el azúcar, en especial la glucosa que es el azúcar que más contiene la tuna (Zenteno et al., 2015, p. 146), la concentración media de glucosa permite indicar que en $240 \mathrm{ml}$ del jugo de los frutos contiene $10 \%$ de la glucosa recomendada para consumo diario (Ibídem, p. 147).

Como hace mención Sumaya, Suárez, Cruz, Alanís \& Sampedro (Op. Cit., 436) en los años de alta producción suele perderse hasta un $60 \%$ de la cosecha debido principalmente a problemas de comercialización, tales como: a) la falta de un mercado asegurado, b) la carencia de espacios de acopio y almacenamiento adecuados para la conservación de la tuna, c) la ausencia de infraestructura de transporte para movilizar el producto y d) la desarticulación de la cadena productiva en donde los procesos de producción y comercialización típicamente se dan por separados.

Además, la mayor inversión que tiene el productor en la comercialización de la tuna es precisamente su almacenamiento, siendo el $40 \%$ de los costos por ser altamente perecedera (dato obtenido por González et al, 2001, citado por Jolapa, Aguilar, Ortiz \& García, 2011, p. 611).

La producción de tuna (Opuntia amyclaea T.) y nopalito (Opuntia spp) puede ser una alternativa para los habitantes de las zonas áridas y semiáridas, pues la mayor superficie de México corresponde a estos ambientes, donde es incosteable la producción de otros cultivos como el maíz, que requieren suelos fértiles y cantidades de agua no disponibles en estas áreas (Callejas et al., 2009, p. 74), "aunque la tuna se distribuye desde Canadá hasta la Argentina y debido a estudios taxonómicos se considera a México como el centro de diseminación de las mismas (Hernández, 1985, p. 1)", pero nuestro país no ha explotado este potencial como debería de ser.

Las tres principales regiones productoras son: la región sur, integrada por el Estado de Puebla y Oaxaca; la región centro, conformada por el Estado de México e Hidalgo; y la región centro norte, integrada por los Estados de Guanajuato, Jalisco, Aguascalientes, San Luis Potosí y Zacatecas. Por Estado, el principal productor es el Estado de México, cuya producción representa el $45.2 \%$, seguido de Zacatecas con el $23.3 \%$, Puebla con el $11.5 \%$ e Hidalgo con el 
ANÁLISIS COMPARATIVO ENTRE UN JARABE DE TUNA (OPUNTIA AMYCLAEA T.) Y UN JARABE COMERCIAL SABOR MAPLE

8.1\% (Ibídem, p. 606). Por lo que el Estado de Hidalgo es uno de los centros productores de tuna más importante del país, ocupando el cuarto lugar nacional. Para el Estado de Hidalgo las dos regiones de mayor producción son el Valle del Mezquital y el Altiplano Hidalguense (Ibídem, p. 607), pero como ya se mencionó, este cultivo no ha rendido las ganancias deseadas por los productores y su economía es cada vez más precaria.

También el mercado internacional de la tuna está restringido a nichos de consumidores con características sociales y culturales bien definidas, son los que muestran una gran lealtad hacia los productos tradicionales de sus países de origen. Particularmente, en los Estados Unidos (en las ciudades de San Antonio, Chicago, Los Ángeles, Miami y Nueva York) y Canadá (en Montreal, Toronto, Edmonton y Vancouver), la demanda de productos típicos o tradicionales, como la tuna, se focaliza en los consumidores mexicanos, latinoamericanos y europeos de origen latino. A pesar de que México es el primer productor mundial de tuna, es decir, México es el país líder en la producción de nopal reportando el 90\% de la producción mundial (Cruz, 2010, p. 3), no ha podido acceder al mercado de otros países, como lo han hecho Italia, Israel, Sudáfrica y Chile (Méndez \& García, Op. Cit., p. 5); y desafortunadamente la agroindustria formal de alimentos en México no se ha interesado en explotar los beneficios de este producto a través de la industrialización (Cruz, Op Cit., p. 3).

La estacionalidad de las cosechas es otro problema, ya que la mayor parte de la tuna se cosecha en 90 días (Sumaya, Suárez, Cruz, Alanís \& Sampedro, Op. Cit., p. 436), aunque existe producción temprana en el mes de mayo y tardía en noviembre (Ayllon, et al. 2015, p. 1617), siendo el periodo más alto de producción en agosto y septiembre; y por lo mismo donde el precio es más bajo por su sobre oferta, ya que llega a ser hasta un $50 \%$ por debajo de su precio promedio (Zegbe \& Mena, 2008, p. 85).

Resulta importante tomar en cuenta algunos factores para que no merme su calidad, por ser la tuna un fruto altamente perecedero, con vida poscosecha que varía entre nueve y quince días a temperatura ambiente, lo cual dificulta su almacenamiento y comercialización (Pinedo, Franco \& Hernández, 2010, p. 43). Por lo que el potencial de producción y lograr obtener los mejores productos del continente es algo que se puede convertir en una ventaja competitiva sostenible con potencial de éxito si se consigue obtener un producto de excelente calidad (Valle \& Vázquez-Bustelo, 2009, p. 62). 
ANÁLISIS COMPARATIVO ENTRE UN JARABE DE TUNA (OPUNTIA AMYCLAEA T.) Y UN JARABE COMERCIAL SABOR MAPLE

Una alternativa viable resulta ser su industrialización in situ, bajo un proceso fácil y de bajo costo; que una de sus características sea el poder alargar su vida de anaquel, es decir, que si en fresco tiene una vida de nueve a quince días al momento de darle un valor agregado en su procesamiento su vida pueda superar los seis meses, logrando con esto no únicamente la estabilidad del producto, sino fabricar un producto donde la comercialización pueda ser en otras latitudes al tener mucho más tiempo para su distribución. Otra de las ventajas que se obtiene es, buscar un producto novedoso que le resulte agradable a los futuros consumidores y también lograr un precio mucho más atractivo que la comercialización del producto en fresco.

Existen otras ventajas que se obtienen con esta industrialización: se reactiva la economía de la zona, ya que se van a utilizar muchas más personas en el proceso y en su comercialización, evitando también con esto los intermediarios, que aprovechan la poca duración del producto en fresco para presionar al productor a su rápida venta.

Al buscar industrializar sólo la producción de tuna que no se logra vender en fresco se vuelve una empresa de desarrollo sustentable, ya que satisface las necesidades de la presente generación sin comprometer la capacidad de las futuras generaciones para que satisfagan sus propias necesidades (Brundtland, citado por Cabañas, 2013, p. 31).

El producto que se busca desarrollar tiene una similitud con otros productos de confitería, que tienen buena aceptación en el mercado (Mijangos, 2015; Rico, 2005), con la ventaja de que la tuna, al no tener que almacenarse a granel, por la necesidad de procesarse lo más pronto posible por su alta humedad, no tiene ataques de alimañas o roedores como es el caso de otras materias primas (PROCEDE, 2005, p. 82).

\section{MATERIAL Y MÉTODO}

Se desarrolló una miel tipo jarabe utilizando como base la tuna, elaborado en la Universidad Autónoma del Estado de Hidalgo, donde ya se han realizado las pruebas de vida en anaquel, es decir, el producto elaborado se tuvo en observación por más de seis meses sin ninguna alternación, tanto física como microbiana.

Para elaborar este producto, como ya se mencionó, se usó la tuna como materia prima, pero también se utilizaron otros insumos, que aportaron alguna característica al producto, como son: 
ANÁLISIS COMPARATIVO ENTRE UN JARABE DE TUNA (OPUNTIA AMYCLAEA T.) Y UN JARABE COMERCIAL SABOR MAPLE

- Azúcar: Son hidratos de carbono que ofrecen dulzor al producto (Quiles, 2013, p. 32).

- Glucosa: Para ayudar a disolver el azúcar y darle cuerpo al producto. Es el principal constituyente de una miel (Sixto \& Knochen, 2011, p. 72).

- Ácido cítrico: Como conservador y mejora de propiedades organolépticas en alimentos (Muñoz, Sáenz, López, Cantú \& Barajas, 2014, p. 21).

- Carboximetilcelulosa (CMC): Es un polímero que actúa como ligante, espesante y estabilizante, además produce materiales transparentes (Valle, López, Reyes, Castillo \& Santos, 2008, p. 236).

- Benzoato de Sodio: Como conservador utilizándolo bajo los rangos permitidos en la Norma Oficial Mexicana NOM (2012, p. 14).

La siguiente etapa, es saber si tiene aceptación por parte de los consumidores potenciales y posteriormente saber dentro de qué línea de productos se le puede ubicar, considerando que quizá sea en los jarabes sabor maple, pero con un nuevo sabor, para realizar un análisis comparativo con el producto similar al desarrollado se utilizó un jarabe que ya existe en el mercado sabor Maple de la marca PROmesa de $300 \mathrm{ml}$ para conocer si existe un grado de asociación (Aguayo, 2007, p. 1) entre ambos por medio de un panel de degustación y por lo tanto conocer si se puede posicionar el producto elaborado dentro de los jarabes de sabor. Para fines de identificación, al producto comercial se le asignó el número 403 y al producto que se desarrolló el 315 (ver figura 1).

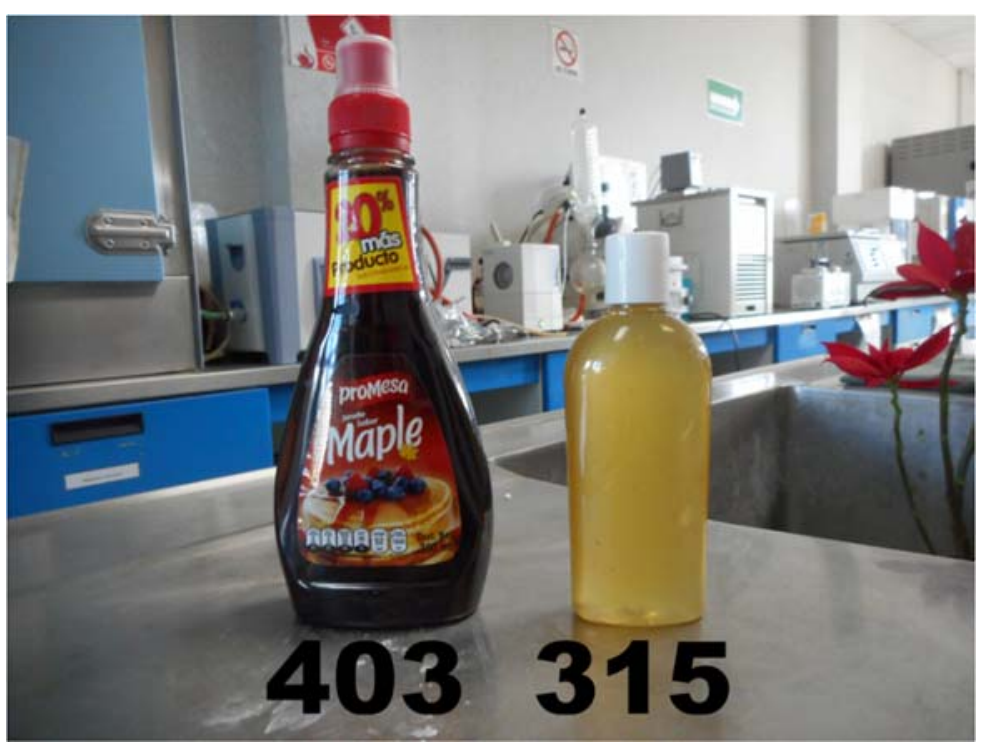

Figura 1. Presentación de ambos productos. 
ANÁLISIS COMPARATIVO ENTRE UN JARABE DE TUNA (OPUNTIA AMYCLAEA T.) Y UN JARABE COMERCIAL SABOR MAPLE

Para que resultara más viable su degustación, se colocó a trozos de hotcakes un ml de producto. A continuación, se muestra en las figuras 2 y 3 las dos alternativas preparadas:

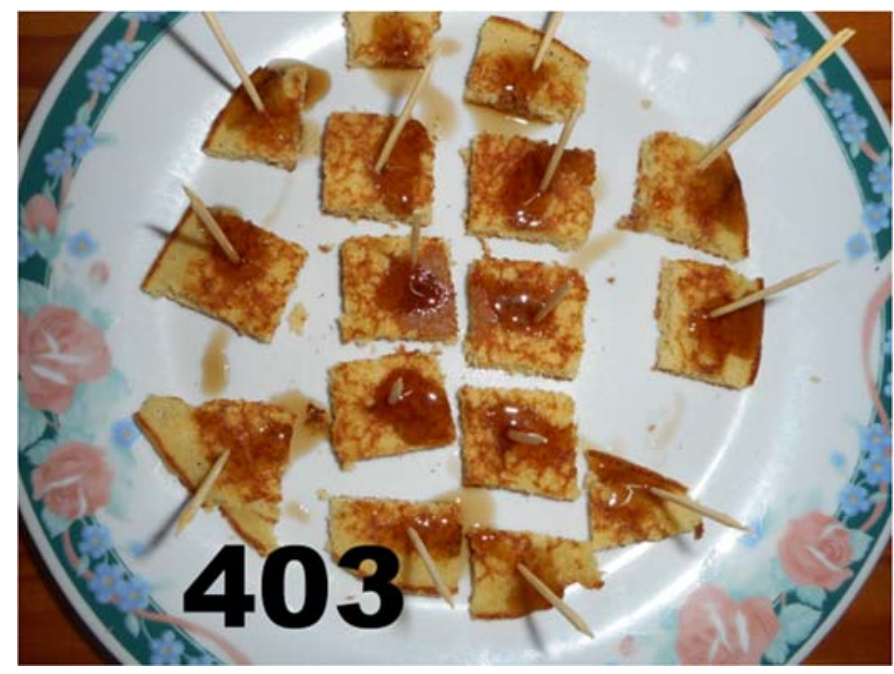

Figura 2. La alternativa 403 donde se utilizó el producto comercial para su degustación.

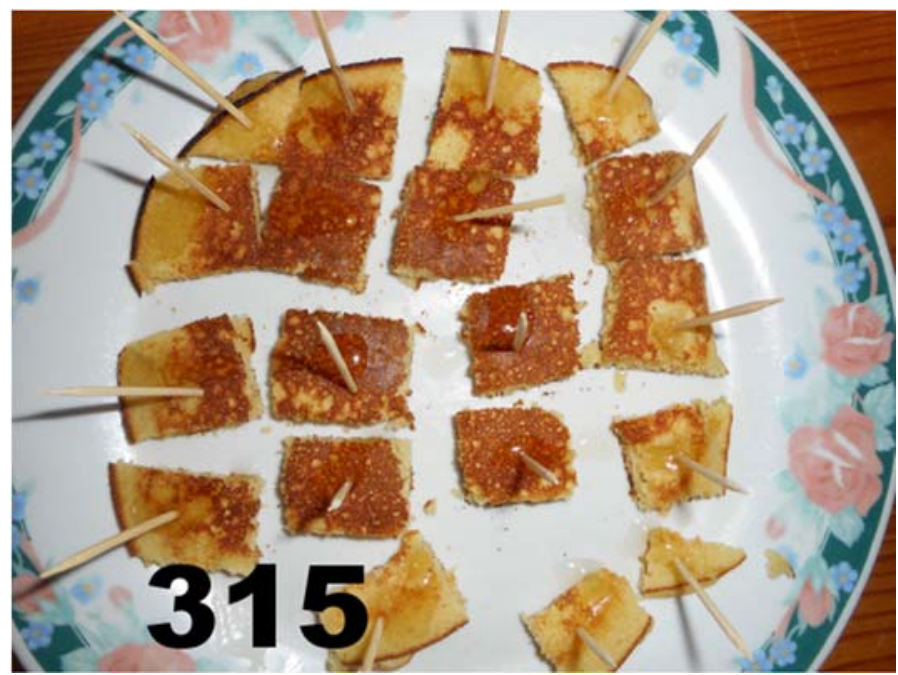

Figura 3. La alternativa 315 donde se utilizó el producto que se desarrolló para su degustación.

Los panelistas, posterior a la degustación de ambos productos, se les proporcionó un instrumento para ser respondido enseguida, las preguntas fueron relacionadas a tres aspectos: 
ANÁLISIS COMPARATIVO ENTRE UN JARABE DE TUNA (OPUNTIA AMYCLAEA T.) Y UN JARABE COMERCIAL SABOR MAPLE

sabor, consistencia y color, por lo que partimos de las siguientes tres hipótesis y sus respectivas hipótesis nulas:

\section{Hipótesis}

- $\mathrm{H}_{1}=$ El producto desarrollado es asociado en sabor al producto comercial.

- $\mathrm{H}_{0}=$ El producto desarrollado no es asociado en sabor al producto comercial.

- $\mathrm{H}_{2}=$ El producto desarrollado es asociado en consistencia al producto comercial.

- $\mathrm{H}_{0}=$ El producto desarrollado no es asociado en consistencia al producto comercial.

- $\mathrm{H}_{3}=$ El producto desarrollado es asociado en color al producto comercial.

- $\mathrm{H}_{0}=$ El producto desarrollado no es asociado en color al producto comercial

Para equilibrar la degustación se alternaron ambos productos en el inicio de la prueba; es decir, los panelistas nones iniciaban con el producto 403 y los panelistas pares con el producto 315.

\section{$t$ de student}

Para comprobar la hipótesis se utilizó la t de student para datos apareados (relacionados), que es cuando para cada observación en un grupo tenemos una observación asociada en el segundo grupo (Barón \& Téllez, s/f: 23); es decir, los mismos que participan en el panel del primer grupo lo hacen también en el segundo. Para los muestreos pequeños se sugiere la $t$ de student: menores de treinta panelistas en nuestro caso (Sánchez, 2015, p. 59).

\section{Número de panelistas y escala}

Para nuestro caso se utilizaron 24 panelistas. A los cuales se les solicitó su valoración en la Escala de Lickert (Hernández-Sampieri, 2003, p. 368), donde las cinco escalas eran: 5) Muy buena, 4) Buena, 3) Aceptable, 2) Mala y 1) Muy mala, utilizando como parámetro el estudio de Barriga, Riofrio, Salas \& Albrecht-Ruíz (2007, p. 87), donde se tiene como límite de rechazo si el promedio de los valores obtenidos es inferior a 3.0, esto con el objeto de también conocer que, aunque no exista grado de asociación entre ambos productos, también saber si existe una aceptación. 
ANÁLISIS COMPARATIVO ENTRE UN JARABE DE TUNA (OPUNTIA AMYCLAEA T.) Y UN JARABE COMERCIAL SABOR MAPLE

\section{Variables}

Las variables de estudio son: sabor, consistencia y color; donde para el caso de la $T$ de Student el valor que se utiliza es la diferencia entre ambos productos (Galindo, 2015), como ya se mencionó, el producto comercial se identifica con el número 403 y el producto desarrollado con el número 315, por lo que los valores obtenidos fueron los que se presentan en la tabla 1.

\section{Tabla 1}

Captura de los valores de los panelistas de las tres dimensiones: sabor, consistencia y color

\begin{tabular}{|c|c|c|c|c|c|c|c|c|c|}
\hline \multicolumn{4}{|c|}{ Sabor } & \multicolumn{3}{|c|}{ Consistencia } & \multicolumn{3}{|c|}{ Color } \\
\hline Panelista & 403 & 315 & DIF. & 403 & 315 & DIF. & 403 & 315 & DIF. \\
\hline 1 & 4 & 3 & 1 & 3 & 3 & 0 & 5 & 3 & 2 \\
\hline 2 & 4 & 4 & 0 & 3 & 4 & -1 & 4 & 2 & 2 \\
\hline 3 & 5 & 5 & 0 & 4 & 4 & 0 & 4 & 1 & 3 \\
\hline 4 & 3 & 4 & -1 & 5 & 5 & 0 & 3 & 1 & 2 \\
\hline 5 & 5 & 4 & 1 & 3 & 3 & 0 & 4 & 2 & 2 \\
\hline 6 & 4 & 4 & 0 & 4 & 5 & -1 & 3 & 2 & 1 \\
\hline 7 & 3 & 4 & -1 & 3 & 2 & 1 & 5 & 3 & 2 \\
\hline 8 & 4 & 3 & 1 & 2 & 3 & -1 & 4 & 2 & 2 \\
\hline 9 & 3 & 2 & 1 & 2 & 5 & -3 & 5 & 4 & 1 \\
\hline 10 & 4 & 3 & 1 & 5 & 4 & 1 & 3 & 2 & 1 \\
\hline 11 & 5 & 2 & 3 & 2 & 3 & -1 & 4 & 2 & 2 \\
\hline 12 & 2 & 2 & 0 & 4 & 4 & 0 & 5 & 3 & 2 \\
\hline 13 & 3 & 3 & 0 & 3 & 4 & -1 & 3 & 1 & 2 \\
\hline 14 & 4 & 3 & 1 & 4 & 5 & -1 & 4 & 2 & 2 \\
\hline 15 & 4 & 4 & 0 & 2 & 3 & -1 & 5 & 3 & 2 \\
\hline 16 & 5 & 3 & 2 & 5 & 4 & 1 & 3 & 2 & 1 \\
\hline 17 & 5 & 4 & 1 & 3 & 4 & -1 & 4 & 2 & 2 \\
\hline 18 & 4 & 3 & 1 & 2 & 3 & -1 & 5 & 3 & 2 \\
\hline 19 & 5 & 4 & 1 & 3 & 4 & -1 & 5 & 4 & 1 \\
\hline 20 & 3 & 3 & 0 & 4 & 5 & -1 & 3 & 2 & 1 \\
\hline 21 & 4 & 2 & 2 & 5 & 4 & 1 & 4 & 2 & 2 \\
\hline 22 & 4 & 3 & 1 & 2 & 3 & -1 & 4 & 3 & 1 \\
\hline
\end{tabular}


ANÁLISIS COMPARATIVO ENTRE UN JARABE DE TUNA (OPUNTIA AMYCLAEA T.) Y UN JARABE COMERCIAL SABOR MAPLE

\begin{tabular}{|c|c|c|c|c|c|c|c|c|c|}
\hline \multicolumn{4}{|c|}{ Sabor } & \multicolumn{3}{|c|}{ Consistencia } & \multicolumn{3}{|c|}{ Color } \\
\hline Panelista & 403 & 315 & DIF. & 403 & 315 & DIF. & 403 & 315 & DIF. \\
\hline 23 & 3 & 4 & -1 & 2 & 4 & -2 & 3 & 3 & 0 \\
\hline 24 & 5 & 4 & 1 & 3 & 4 & -1 & 5 & 4 & 1 \\
\hline Promedio & 3.96 & 3.33 & & 3.25 & 3.83 & & 4.04 & 2.42 & \\
\hline
\end{tabular}

Fuente: Elaboración propia.

Sabor. Con estos datos se corre en el paquete estadístico SPSS los valores del sabor, obteniéndose los siguientes resultados que se presentan en la figura 4.

\begin{tabular}{|c|c|c|c|c|c|c|c|c|c|}
\hline \multicolumn{10}{|c|}{ Prueba de muestras relacionadas } \\
\hline \multirow{3}{*}{\multicolumn{2}{|c|}{ 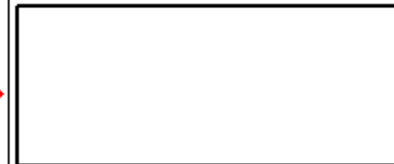 }} & \multicolumn{5}{|c|}{ Diferencias relacionadas } & \multirow[b]{3}{*}{$t$} & \multirow[b]{3}{*}{$\mathrm{gl}$} & \multirow{3}{*}{ Sig. (bilateral) } \\
\hline & & \multirow[b]{2}{*}{ Media } & \multirow[b]{2}{*}{$\begin{array}{l}\text { Desviación } \\
\text { típ. }\end{array}$} & \multirow[b]{2}{*}{$\begin{array}{l}\text { Error típ de la } \\
\text { media }\end{array}$} & \multicolumn{2}{|c|}{$\begin{array}{l}\text { 95\% Intervalo de confianza para } \\
\text { la diferencia }\end{array}$} & & & \\
\hline & & & & & Inferior & Superior & & & \\
\hline Par 1 & $\begin{array}{l}\text { Sabor (Prod. 403) - Sabor } \\
\text { (Prod. 315) }\end{array}$ & .45833 & .88363 & .18037 & .08521 & .83146 & 2.541 & 23 & .018 \\
\hline
\end{tabular}

Figura 4. Cálculo de la significancia del sabor con SPSS.

Donde la significación, el p-valor, es menor a 0.05, ya que como se visualiza en la figura 4, su valor es de 0.018; por tal motivo, con lo que respecta a la hipótesis planteada $\mathrm{H}_{1}$, se acepta, para corroborarlo se capturaron los valores obtenidos en una calculadora estadística en línea (http://www.conexionismo.com/calculadoras_estadisticas/) (ver figura 5).

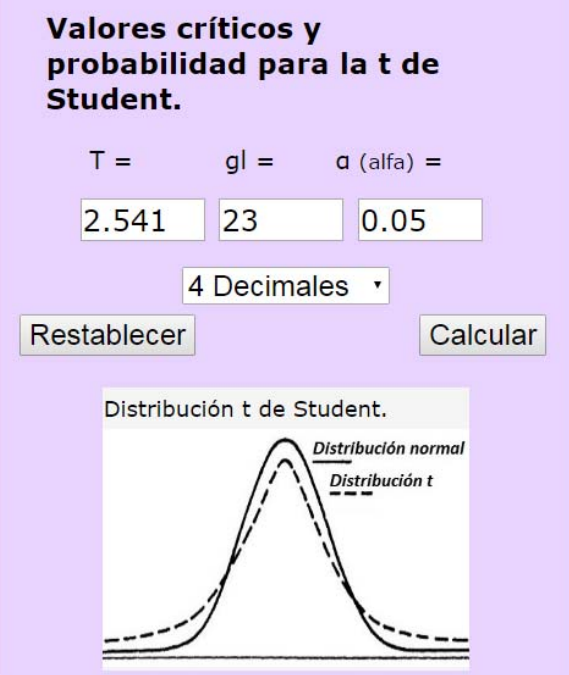

Figura 5. La distribución de la $t$ de student para el sabor. 
ANÁLISIS COMPARATIVO ENTRE UN JARABE DE TUNA (OPUNTIA AMYCLAEA T.) Y UN JARABE COMERCIAL SABOR MAPLE

En mismo software tiene una alternativa que se llama decisión, la cual al aplicarlo indica si se rechaza o no la hipótesis, para nuestro caso se rechaza como aparece en la figura 6.

\section{Decisión por p-valor: \\ Para 1 cola: $0.05 \geq 0.0091$ \\ Se rechaza la $\mathrm{H}_{0}$ \\ Para 2 colas: $0.025 \geq 0.0091$ \\ Se rechaza la $\mathrm{H}_{0}$}

Figura 6. Decisión de p-valor donde se rechaza la hipótesis nula del sabor.

Para ambos casos (una o dos colas) se rechaza la hipótesis nula, por lo que se puede afirmar, como ya se obtuvo al aplicar los valores en el software del SPSS, que los panelistas si asociaron el producto desarrollado con el comercial con lo que respecta al sabor.

Consistencia. Con estos datos se corre en el paquete estadístico SPSS los valores de la consistencia obteniéndose los siguientes resultados que se presentan en la figura 7.

\begin{tabular}{|c|c|c|c|c|c|c|c|c|c|}
\hline & & \multicolumn{5}{|c|}{ Diferencias relacionadas } & \multirow[b]{3}{*}{$t$} & \multirow[b]{3}{*}{ gl } & \multirow{3}{*}{ Sig. (bilateral) } \\
\hline & & \multirow[b]{2}{*}{ Media } & \multirow{2}{*}{$\begin{array}{l}\text { Desviación } \\
\text { típ. }\end{array}$} & \multirow{2}{*}{$\begin{array}{l}\text { Error típ de la } \\
\text { media }\end{array}$} & \multicolumn{2}{|c|}{$\begin{array}{l}95 \% \text { Intervalo de confianza para } \\
\text { la diferencia }\end{array}$} & & & \\
\hline & & & & & Inferior & Superior & & & \\
\hline Par 1 & $\begin{array}{l}\text { Consistencia (Prod. 403) } \\
\text { - Consistencia (315) }\end{array}$ & -.58333 & .97431 & .19888 & -.99475 & -.17192 & -2.933 & 23 & .007 \\
\hline
\end{tabular}

Figura 7. Cálculo de la significancia de la consistencia con SPSS.

Donde la significación, el p-valor, es menor a 0.05, ya que como se visualiza en la figura 7, su valor es de 0.007; por tal motivo con lo que respecta a la hipótesis planteada $\mathrm{H}_{2}$, se acepta, para corroborarlo se capturaron los valores obtenidos en una calculadora estadística en línea (http://www.conexionismo.com/calculadoras_estadisticas/) (ver figura 8). 
ANÁLISIS COMPARATIVO ENTRE UN JARABE DE TUNA (OPUNTIA AMYCLAEA T.) Y UN JARABE COMERCIAL SABOR MAPLE

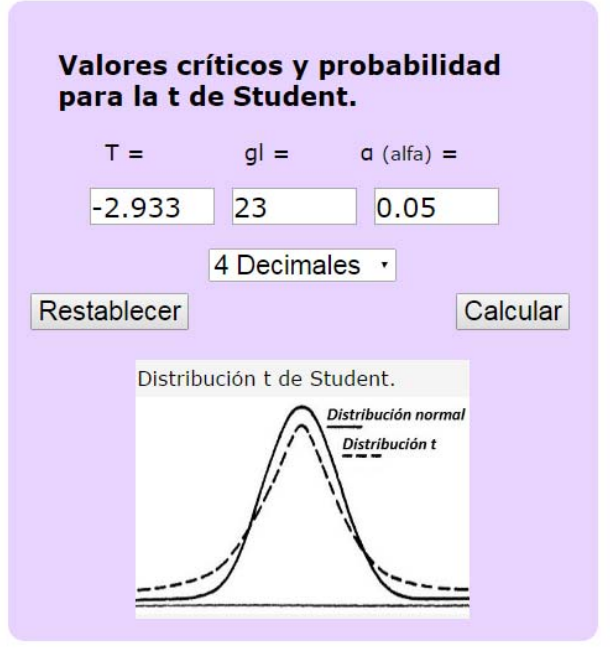

Figura 8. La distribución de la $t$ de student para la consistencia.

En mismo software tiene una alternativa que se llama decisión, la cual al aplicarlo indica si se rechaza o no la hipótesis, para nuestro caso se rechaza como aparece en la figura 9.

\section{Decisión por p-valor: \\ Para 1 cola: $0.05 \geq 0.0037$ \\ Se rechaza la $\mathrm{H}_{0}$ \\ Para 2 colas: $0.025 \geq 0.0037$ \\ Se rechaza la $\mathrm{H}_{0}$}

Figura 9. Decisión de p-valor donde se rechaza la hipótesis nula de la consistencia.

Para ambos casos (una o dos colas) se rechaza la hipótesis nula, por lo que puede afirmarse, como ya se obtuvo al aplicar los valores en el software del SPSS, que los panelistas si asociaron el producto desarrollado con el comercial con lo que respecta a la consistencia.

Color. Con estos datos se corre en el paquete estadístico SPSS los valores del color, obteniéndose los siguientes resultados que se presentan en la figura 10. 
ANÁLISIS COMPARATIVO ENTRE UN JARABE DE TUNA (OPUNTIA AMYCLAEA T.) Y UN JARABE COMERCIAL SABOR MAPLE

\begin{tabular}{|c|c|c|c|c|c|c|c|c|c|}
\hline & & \multicolumn{5}{|c|}{ Diferencias relacionadas } & \multirow[b]{3}{*}{$t$} & \multirow[b]{3}{*}{$\mathrm{gl}$} & $0.000<0.05$ \\
\hline & & \multirow[b]{2}{*}{ Media } & \multirow[b]{2}{*}{$\begin{array}{l}\text { Desviación } \\
\text { tip. }\end{array}$} & \multirow[b]{2}{*}{$\begin{array}{l}\text { Error típ de la } \\
\text { media }\end{array}$} & \multicolumn{2}{|c|}{$\begin{array}{l}95 \% \text { Intervalo de confianza para } \\
\text { la diferencia }\end{array}$} & & & \\
\hline & & & & & Inferior & Superior & & & Sig. (bilateral) \\
\hline Par 1 & $\begin{array}{l}\text { Color (Prod. 403) - Color } \\
\text { (Prod. 315) }\end{array}$ & 1.62500 & .64690 & .13205 & 1.35184 & 1.89816 & 12.306 & 23 & .000 \\
\hline
\end{tabular}

Figura 10. Cálculo de la significancia del color con SPSS.

Donde la significación, el p-valor, es menor a 0.05 , ya que como se visualiza en la figura 10, su valor es de 0.000 , quizá en el cuarto o quinto decimal aparezca un valor diferente de cero; por tal motivo con lo que respecta a la hipótesis planteada $\mathrm{H}_{3}$, se acepta, para corroborarlo se capturaron los valores obtenidos en una calculadora estadística en línea (http://www.conexionismo.com/calculadoras_estadisticas/) (ver figura 11).

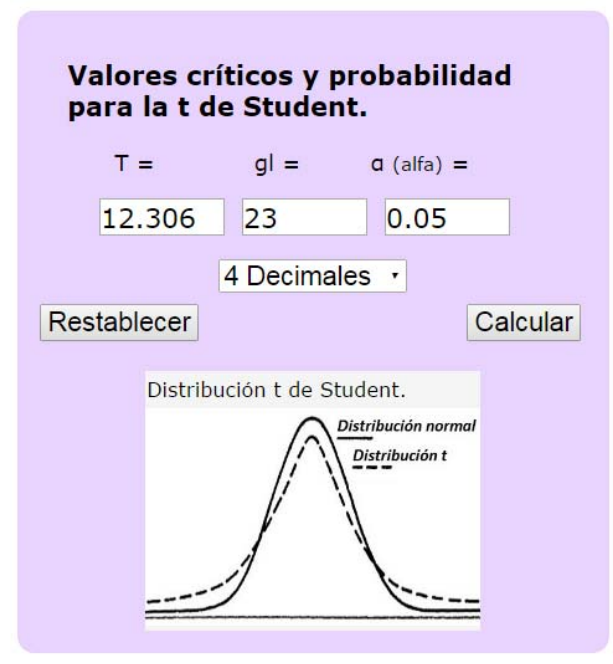

Figura 11. La distribución de la t de student para el color.

En mismo software tiene una alternativa que se llama decisión, la cual al aplicarlo indica si se rechaza o no la hipótesis, para nuestro caso se rechaza como aparece en la figura 12. 


\section{Decisión por p-valor: \\ Para 1 cola: $0.05 \geq 0$ \\ Se rechaza la $\mathrm{H}_{0}$ \\ Para 2 colas: $0.025 \geq 0$ \\ Se rechaza la $\mathrm{H}_{0}$}

Figura 12. Decisión de p-valor donde se rechaza la hipótesis nula del color.

Para ambos casos (una o dos colas) se rechaza la hipótesis nula, por lo que se puede afirmar, como ya se obtuvo al aplicar los valores en el software del SPSS, que los panelistas si asociaron el producto desarrollado con el comercial con lo que respecta al color.

Por otro lado, los valores obtenidos en la Escala de Lickert nos indican, como se mencionó anteriormente, existen valores altos al no ser sus promedios inferiores a 3.0 (Barriga, Riofrio, Salas \& Albrecht-Ruíz, 2007, p. 87) a excepción del color en el producto desarrollado (315), que como se puede percibir en las fotos su tono es un amarillo muy transparente y quizá no es un color que resulte agradable a la vista de los panelistas, por lo que esto puede desencadenar otro estudio.

\section{Fiabilidad}

La confiabilidad se define como el grado en que un instrumento de varios ítems mide consistentemente una muestra de población. La medición consistente se refiere al grado en que una medida está libre de errores (Oviedo \& Campos-Arias, 2005, pp. 573-574). Por lo que el coeficiente Alfa de Cronbach, es un índice usado para medir la confiabilidad del tipo consistencia interna de una escala, es decir, para evaluar la magnitud en que los ítems de un instrumento están correlacionados. En otras palabras, el Alfa de Cronbach es el promedio de las correlaciones entre los ítems que son parte de un instrumento (Ibídem, p. 575).

Los valores de este coeficiente oscilarán entre 0 y 1 y únicamente se obtendrán valores con signo menos si la relación entre los ítems es negativa, en cuyo caso no procedería plantearse la 
ANÁLISIS COMPARATIVO ENTRE UN JARABE DE TUNA (OPUNTIA AMYCLAEA T.) Y UN JARABE COMERCIAL SABOR MAPLE

posibilidad de calcular un índice de fiabilidad de la escala (Visauta \& Martori, 2003, p. 296). Para nuestro caso los valores de los tres grupos de ítems (sabor, consistencia y color) tienen un valor positivo como se puede apreciar en las siguientes impresiones de pantalla de los resultados del coeficiente de Alfa de Cronbach.

Para el caso del sabor el valor del Alfa de Cronbach fue de 0.6469, (ver figura 13).

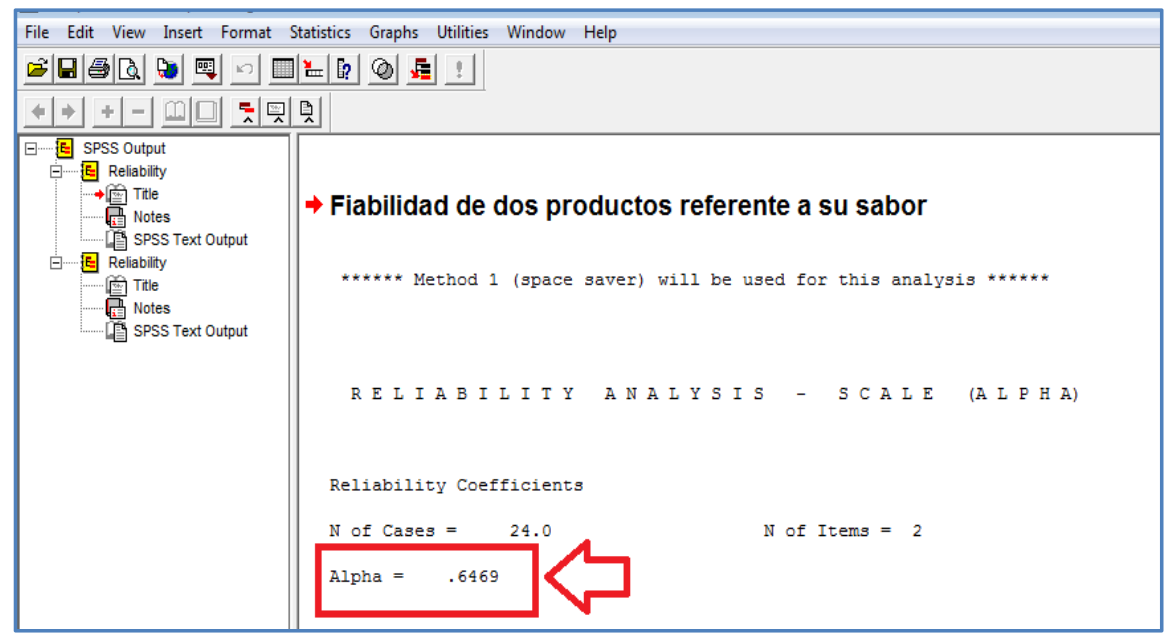

Figura 13. Valor del Alfa de Cronbach en el sabor.

Con lo que respecta a la consistencia el valor del Alfa de Cronbach fue de 0.6469, (ver figura 14).

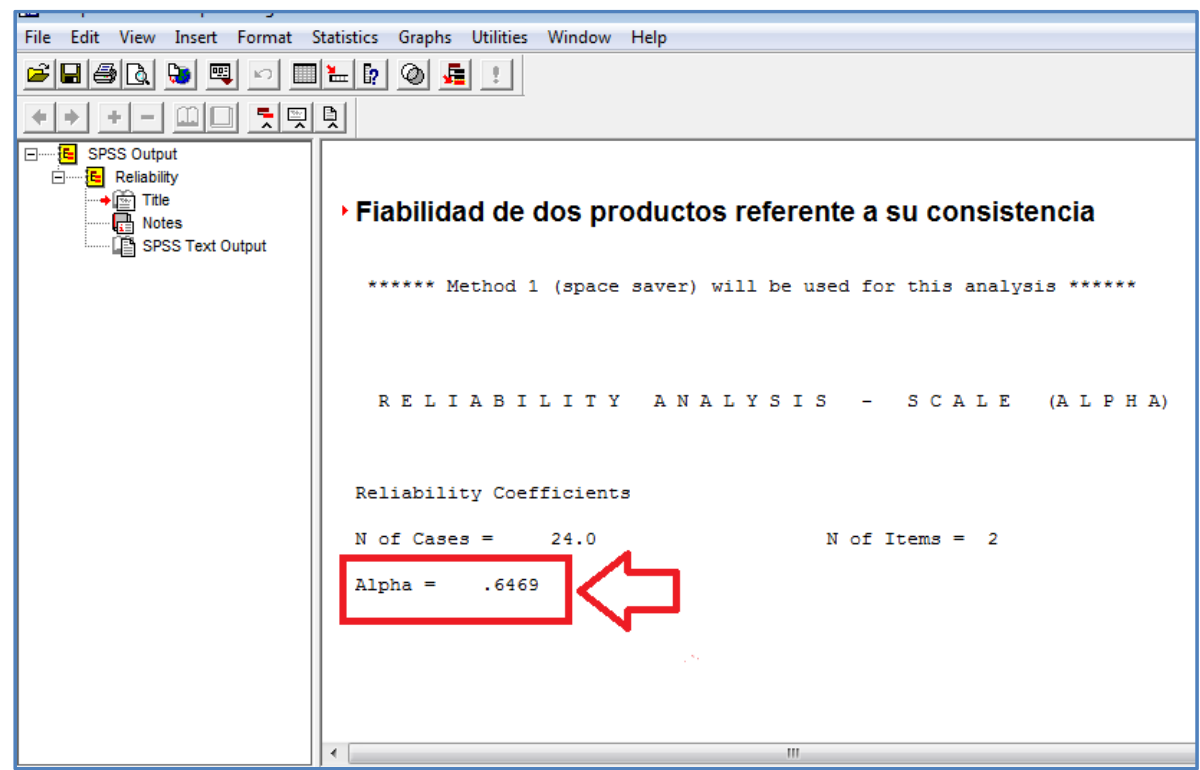

Figura 14. Valor del Alfa de Cronbach en la consistencia. 
ANÁLISIS COMPARATIVO ENTRE UN JARABE DE TUNA (OPUNTIA AMYCLAEA T.) Y UN JARABE COMERCIAL SABOR MAPLE

Por último, para el caso del color el valor del Alfa de Cronbach fue de 0.8280, (ver figura 15).

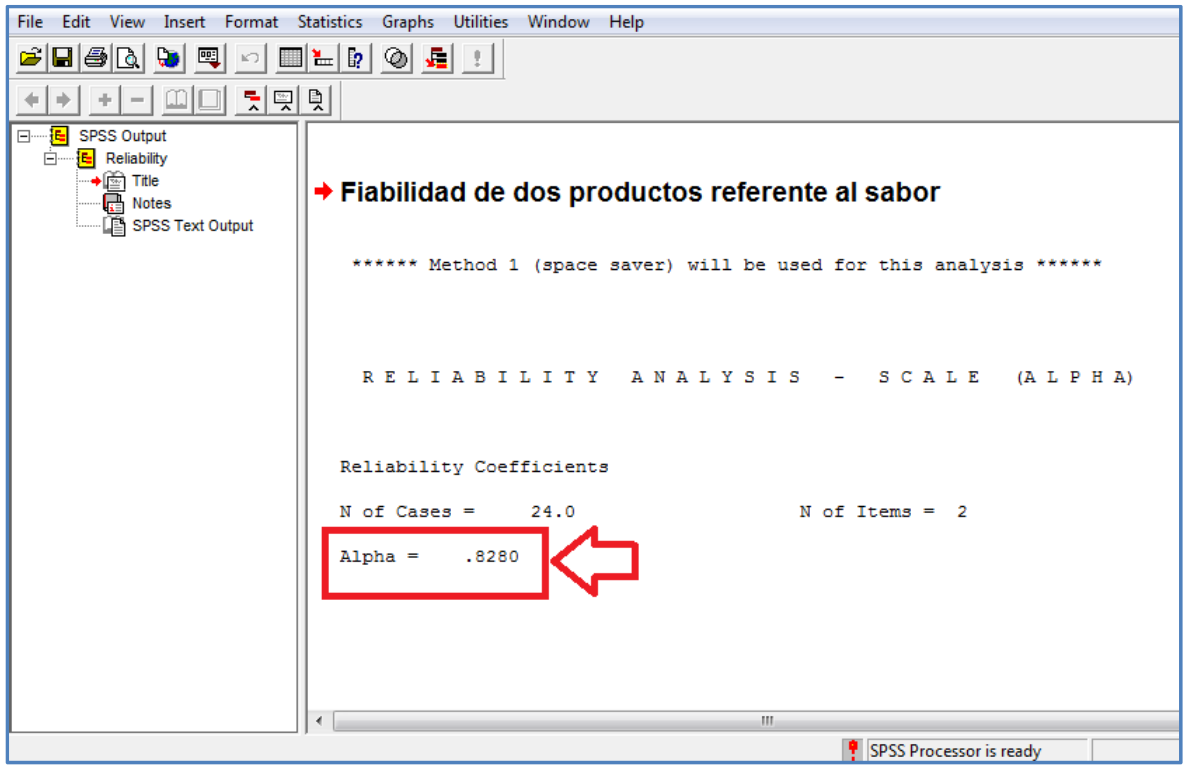

Figura 15. Valor del Alfa de Cronbach en el color.

\section{RESULTADOS}

En las tres hipótesis planteadas sobre: sabor, consistencia y color existió una asociación entre el producto comercial y nuestra propuesta, por lo que se presume que los panelistas percibieron el producto presentado como uno más de la línea de jarabes de sabores, por tal motivo resulta positivo posicionar este producto dentro de esta gama.

Con lo que respecta a los valores obtenidos en la degustación por medio de la Escala de Lickert, donde los rangos son de 1 a 5, siendo el de mayor agrado 5, si existió aceptación del producto en dos de las tres dimensiones, rebasando al producto comercial en el caso de la consistencia, ya que la alternativa comercial tuvo 3.25 y nuestra propuesta 3.83 , con lo que respecta al sabor nuestra alternativa tuvo un valor menor, ya que fue de 3.33 y la comercial 3.96, pero desde el punto de vista de Barriga, Riofrio, Salas \& Albrecht-Ruíz (2007, p. 87), estos valores son aceptables al ser mayores de 3.0. Sólo en el caso del color su valor fue de 2.42, por lo que no se considera aceptado por los panelistas, ya que el único valor crítico del producto desarrollado fue su color, una alternativa es agregarle al producto una amplia gama de colores y conocer la aceptación por medio de otra degustación que daría pie a una nueva investigación. 
ANÁLISIS COMPARATIVO ENTRE UN JARABE DE TUNA (OPUNTIA AMYCLAEA T.) Y UN JARABE COMERCIAL SABOR MAPLE

Este trabajo da pauta no sólo para generar alternativas de color, sino también variar el sabor 0 cambiarlo con otros productos y ver si se incrementa su aprobación.

\section{CONCLUSIONES}

En esta propuesta se tiene la gran ventaja del ganar-ganar; es decir, se benefician todas las partes. Como hace mención Cruz (2015, pp. 35-36) cuando cita a Rubio (2013) y Torres (2006): "La producción agrícola es fundamental para un sistema alimentario, cuando éste es frágil, profundiza la pobreza, la desnutrición y el descontento en el medio rural. Por lo que salvaguardar la seguridad alimentaria se vuelve un factor estratégico, ya que se convierte en una amenaza que propicia una degradación de la calidad de vida de los habitantes". Esta propuesta puede generar una estabilidad a los productores de la materia prima del producto que se estudió que es la tuna, ya que se les puede transferir la tecnología de un producto que se ha probado su aceptación y sobre todo es de fácil elaboración y poca inversión para ponerlo en marcha.

Como recomendación a futuro se busca que se desarrolle el plan de negocios, así como la presentación que deberá tener el producto en cuanto a envase y etiquetado. Aunado a esto, es importante filmar el proceso ya que además de capacitarlos en su elaboración los productores tendrán el video de cómo se fabrica paso a paso para que lo tengan presente.

\section{REFERENCIAS BIBLIOGRÁFICAS}

Aguayo, M. (2007). Cómo utilizar "paso a paso" un contraste de hipótesis con SPSS para Windows y alternativamente con EPIINFO y EPIDAT. fabis.org. Dot. Num. 0702004. (p. 1). España: Fundación Andaliza Betuaria para la Investigación en Salud.

Anaya, M. \& Bautista, R. (2008). El nopal forrajero en México: Del siglo XVI al Siglo XX. (Spanish). Revista Agricultura, Sociedad y Desarrollo, 5(2), p. 168.

Ayllon, J., Omaña J., Sangerman, D., Garza, L., Quintero, J. \& Gonzalez, F. (2015). Modelo de transporte en México para la minimización de costos de distribución de tuna (Opuntia spp) en fresco. (Spanish). Revista Mexicana de Ciencias Agrícolas. Instituto Nacional de Investigaciones Forestales, Agrícolas y Pecuarias, 6(7), p. 1617. 
ANÁLISIS COMPARATIVO ENTRE UN JARABE DE TUNA (OPUNTIA AMYCLAEA T.) Y UN JARABE COMERCIAL SABOR MAPLE

Barriga, M., Riofrio, C., Salas, A. \& Albrecht-Ruíz, M. (2007). Cambios bioquímicos y sensoriales en Gamitana (Colossoma macropomum) Fresca almacenada a tres diferentes temperaturas. (Spanish). Revista Bol Investigación, (8), p. 87.

Barón F. \& Téllez F. (s/f). Diferencias que presenta una variable numérica entre dos grupos. Apuntes de Bioestadística. Universidad de Málaga, España. Recuperado de: https://www.bioestadistica.uma.es/baron/apuntes/ficheros/cap04.pdf.

Barrios, M. (2010). Modelo de Negocio. Paraguay: Escuela de Negocios. Universidad Americana.

Cabañas, A. (2013). Financiamiento para una empresa de giro industrial a través de una SOFOM, bajo el enfoque del desarrollo sustentable (Tesis de Maestría). Escuela Superior de Comercio y Administración. Instituto Politécnico Nacional, México.

Callejas, N., Matus J., Garcia J., Martinez M. \& Salas M. (2009). Situación actual y perspectivas de mercado para la tuna, el nopalito y derivados en el estado de México. (Spanish). Revista Agrociencia, 43(1), p. 74.

Cruz, M. (2015). La pyme, el eslabón entre la sustentabilidad y el agricultor: México. (Spanish). Revista Emprendedores de la UNAM, (155), pp. 35-36.

Cruz, R. (2010). Propuesta de un plan de negocios para la industrialización del nopal. Caso Nopalxolco. (Tesis de Maestría). Escuela Superior de Comercio y Administración, Instituto Politécnico Nacional. México.

Galindo, P. (2015). Curso: Estadística para Investigadores. Universidad de Salamanca, España. Recuperado de: http://miriadax.net.

Hernández, D. (1985). Elaboración de vino a partir de la tuna. (Tesis de licenciatura para obtener el título de Ingeniero en Alimentos). Departamento de Biotecnología. Universidad Autónoma metropolitana Unidad Iztapalapa, México, D. F.

Hernández, R., Fernández C., Baptista P., (2003). Metodología de la investigación (3ª. ed.). México: Mc Graw-Hill Interamericana.

Jolapa, J., Aguilar A., Ortiz, O. \& García, L. (2011). Producción y comercialización de tuna en fresco bajo diferentes modalidades en Hidalgo, México. (Spanish). Revista Mexicana de Agronegocios, $X V(28)$, p. 606. 
ANÁLISIS COMPARATIVO ENTRE UN JARABE DE TUNA (OPUNTIA AMYCLAEA T.) Y UN JARABE COMERCIAL SABOR MAPLE

Lerma, A. (2001). Guía para el desarrollo de productos. Un enfoque global. México: Thompson Learning.

Mejía, J. (2013). Enfoca tu plan de marketing en la creatividad. (Spanish). Revista Entrepreneur. 21(10), p. 19.

Méndez, S. \& García, J. (2006). La tuna: producción y diversidad. (Spanish). Boletín BioDiversitas, (68), 4.

Mijangos V. (2015). Dulce de tamarindo con toque artesanal. Revista electrónica: La Nueva Llave Empresarial. Recuperada de: http://www.llaveempresarial.com.mx/2015/11/18/dulcede-tamarindo-con-toque-artesanal/

Muñoz, A., Sáenz, A., López, L., Cantú, L. \& Barajas, L. (2014). Ácido Cítrico: compuesto interesante. Acta Química Mexicana. Revista Científica de la Universidad Autónoma de Coahuila. 6(12), p. 21.

NOM. (2012). Norma Oficial Mexicana. Acuerdo en el que se determinan los aditivos y coadyuvantes en alimentos, bebidas y suplementos alimenticios, su uso y disposición. Publicado el 16 de julio de 2012 en el Diario Oficial. México.

Oviedo, H. \& Campos, A. (2005). Metodología de investigación y lectura crítica de estudios: Aproximación al uso del coeficiente alfa de Cronbach. (Spanish). Revista Colombiana de Psiquiatría, XXXIV(4), pp. 573-575.

Pinedo J. Franco A. \& HERNÁNDEZ, A. (2010). Comportamiento poscosecha de cultivares de tuna por efecto del manejo del huerto y temperatura de frigoconservación. (Spanish). Revista Iberoamericana de Tecnología Poscosecha, 11(1), 43.

PROCEDE. (2005). Estudio de mercado para identificar el potencial de exportación de dulces de leche en el mercado hispano del medio oeste de los Estados Unidos. Secretaría de Economía. México.

Quiles, J. (2013). Patrón de consumo e ingestas recomendadas de azúcar. Revista Nutrición Hospitalaria, 28(4), julio de 2013. Grupo Aula Médica. Madrid, España.

Rico, E. (2005). La dulce Trayectoria de Sonric's de Geniat Innovación. Revista Industria Alimenticia. Recuperado de: http://www.industriaalimenticia.com/articles/85311-la-dulcetrayectoria-de-sonric-s-de-geniat-innovacion . 
ANÁLISIS COMPARATIVO ENTRE UN JARABE DE TUNA (OPUNTIA AMYCLAEA T.) Y UN JARABE COMERCIAL SABOR MAPLE

Sánchez, T. (2015). T de Student: Usos y Abusos. (Spanish). Revista Mexicana de Cardiología, 26(1), p. 59.

Sixto, A. \& Knochen M. (2011). Determinación de glucosa en miel mediante sistemas en flujo de inyección secuencial (SIA) y multiconmutado. Revista Avances en Ciencias e Ingeniería, 2(1). Executive Business School. La Serena, Chile.

Sumaya, M., Suárez, T., Cruz, N., Alanís, E. \& Sampedro, J. (2010). Innovación de productos de alto valor agregado a partir de la tuna mexicana. (Spanish). Revista Mexicana de Agronegocios. Sociedad Mexicana de Administración Agropecuaria, XIV(27), pp. 436-438.

Tomas G., Huamán J., Aguirre R., Bravo M., León J., Guerrero M., Orihuela C., Aviles R. \& Yanqui E. (2012). Estudio químico y fitoquímico de la Opuntia Ficus-indica "tuna", y elaboración de un alimento funcional. (Spanish). Revista de Química e Ingeniería Química, 15(1), p. 70.

Valle, S., López O., Reyes M., Castillo J. \& Santos A. (2008). Recubrimiento comestible basado en goma arábiga y carboximetilcelulosa para conservar frutas en atmósfera modificada. Revista Chapingo serie Horticultura, 14(3), p. 236.

Valle, S. \& Vázquez, D. (2009). Inclusión de los proveedores en el proceso de desarrollo de nuevos productos: Un análisis de la Industria Española. (Spanish). Universia Business Review. Cuarto Trimestre, 62.

Visauta, B. \& Martori, J. (2003). Análisis estadístico con SPSS para Windows. Volumen II. Estadística Multivariante. España: Mc Graw-Hill.

Zegbe, J. \& Mena J. (2008). Retraso de la cosecha en nopal tunero cv. Cristalina. (Spanish). Revista Chapingo serie Horticultura, 14(1), p. 85.

Zenteno, G., Juárez, B., Aguirre, J., Ortíz, M., Zamora, C. \& Rendo, J. (2015). Evaluación de azúcares y fibra soluble en el jugo de variantes de tunas (Opuntia spp). (Spanish). Revista Agrociencia, 49(2), pp. 146-147. 